Supporting Information for

\title{
Light-designed Shark Skin-mimetic Surfaces
}

Wonhee Jo, ${ }^{\dagger}$ Hong Suk Kang, ${ }^{\S}$ Jaeho Choi, ${ }^{\dagger}$ Jinkwan Jung, ${ }^{\dagger}$ Jonghyun Hyun, ${ }^{\dagger}$

Jaehyung Kwon, ${ }^{\dagger}$ Ilju Kim, ${ }^{\dagger}$ Hongkyung Lee, ${ }^{\Uparrow}$ and Hee-Tak Kim ${ }^{*},+, \neq$

${ }^{\dagger}$ Department of Chemical and Biomolecular Engineering, KAIST, Daejeon, 34141, Republic of Korea

KAIST Institute for the NanoCentury, KAIST, Daejeon, 34141, Republic of Korea

Interface Materials and Chemical Engineering Research Center, Korea Research Institute of Chemical Technology (KRICT), Daejeon, 34114, Republic of Korea

`Department of Energy Science and Engineering, DGIST, Daegu, 42988, Republic of Korea

*Correspondence to: heetak.kim@kaist.ac.kr (prof. Hee-Tak Kim) 
Experimental Section/Methods

Fig. S1. Schematic illustration of the optical instruments setup.

Fig. S2. Chemical structure of poly (disperse orange) 3 (PDO3).

Fig. S3. SEM images showing directional mass migration of the azopolymer

Fig. S4. Plot of the transmission for the azopolymer film as a function of the film thickness and the experimentally measured permittivity of azopolymers

Fig. S5. SEM image of a slant interference light-irradiated pillar that has a diameter of 1 or 20 $\mu \mathrm{m}$.

Fig. S6. SEM images of shark skin mimicked surfaces with various denticle densities.

Fig. S7. Photo and SEM image of large-area fabrication and flexible shark skin-mimetic surfaces

Fig. S8. Schematic illustration of the structure transfer process and SEM image of the transferred PUA shark skin-mimetic structure

Fig. S9. SEM image of PS beads used in the ex-situ analysis of the antifouling effect.

Fig. S10. SEM images showing the direction dependency of the antifouling effect. 


\section{Experimental Section/Methods}

Preparation of the micro-pillar array on the azopolymers.

A PDMS mold with an array of cylindrical holes was prepared with a silicon master template containing an array of cylindrical posts with a diameter of $5 \mu \mathrm{m}$, a depth of $5 \mu \mathrm{m}$ and a

spacing of $5 \mu \mathrm{m}$. The surface of the silicon master was treated with tridecafluoro-1,1,2,2-

tetrahydrooctyl-1-trichlorosilane, on which a mixture of a prepolymer and a cross-linker (Sylgard 184, Dow Corning, Midland, MI) at a weight ratio of 10:1 was gently poured and cured at $70{ }^{\circ} \mathrm{C}$ for $5 \mathrm{~h}$; the mixture was degassed by vacuum suction before use. The PDMS mold was then released from the silicon master. The azopolymer, PDO3, with a molecular weight of $4700 \mathrm{~g} \mathrm{~mol}^{-1}$ and a polydispersity index of 1.74 , was synthesized by a solid-state step polymerization of $5.8 \mathrm{mmol}$ disperse orange 3 (Sigma-Aldrich) and $5.8 \mathrm{mmol}$ bisphenol A diglycidyl ether (Sigma-Aldrich), as previously described elsewhere. ${ }^{1}$ The synthesized PDO3 was dissolved in cyclohexanone at $30 \mathrm{w} / \mathrm{w} \%$, and a drop of the solution was spread on a glass substrate. The PDMS mold was then placed on the cast. The holes in the PDMS mold were completely filled with the solution by capillary action. After the solvent was fully evaporated at $60^{\circ} \mathrm{C}$ for $12 \mathrm{~h}$, the PDMS mold was gently removed from the substrate, yielding the array of cylindrical pillars on the glass substrate. The polyurethane acrylate (PUA) materials were purchased from Minuta Tech, Kirea and used throughout the experimnets without post treatment.

$\underline{\text { Irradiation of polarized light on the azopolymer pillar arrays. }}$

A $532 \mathrm{~nm}$ wavelength laser (diode pump solid-state, Melles Griot) with an output power of 20 $\mathrm{mW}$ was split with beam splitter and circularly polarized using a set of half- and quarter-wave plates (as shown in Figure S1, Supporting Information). The intensity of the beam was 
controlled by neutral density filters. The array of pillars was irradiated at an incidence angle. The light irradiation time was precisely controlled with an electronic shutter. Simulation of the field-gradient of the light intensity and absorption in PDO3. For Finite-Different Time-Domain (FDTD) simulations (Lumerical, Ansys, USA), the model structure, which is equivalent to the pillar used, was meshed at $10 \mathrm{~nm}$ along all directions. The permittivity of PDO3, a parameter for the simulations, was experimentally measured with ellipsometry (EP3, Nanofilm).

$\underline{\text { Structural characterizations and surface properties of shark skin-mimetic surfaces. }}$

The structures were investigated using a scanning electron microscope (SEM) (FEI, SIRION, USA) at $15 \mathrm{keV}$. The samples were made conductive by a coating of $10 \AA$ platinum prior to the SEM analysis. The surface topography and height line profiles were investigated using atomic force microscopy (AFM) (XE7, Park Systems, KOR). The water contact angle was measured using a goniometer (DSA 10-Mk2, KRUSS, Germany) for which $3.3 \mu \mathrm{L}$ drops were deposited using a computer-controlled syringe pump.

\section{Ex-situ analysis of the anti-fouling effect.}

The polystyrene (PS) beads were dispersed in water with concentration of $3 \mathrm{wt} \%$. The diameter of PS beads was $50 \mathrm{~nm}$. The shark skin-mimetic surface, which light irradiation condition: (t, $\theta, \varphi, \psi)=(3 \mathrm{~min}, 12 \mathrm{o}, 15 \mathrm{o}, 0 \mathrm{o})$, was cut into size of $2 \times 2 \mathrm{~cm} 2$. The water flowed over the surface with constant flow speed of $3 \mathrm{ml} / \mathrm{sec}$ during 60 seconds. The surface then completely dried in $60 \mathrm{oC}$ oven for 3 hours.

\section{REFERENCES}

1. Kim, M.-J.; Yoo, S.-J.; Kim, D.-Y. A supramolecular chiroptical switch using an amorphous azobenzene polymer. Adv. Funct. Mater. 2006, 16, 2089-2094. 


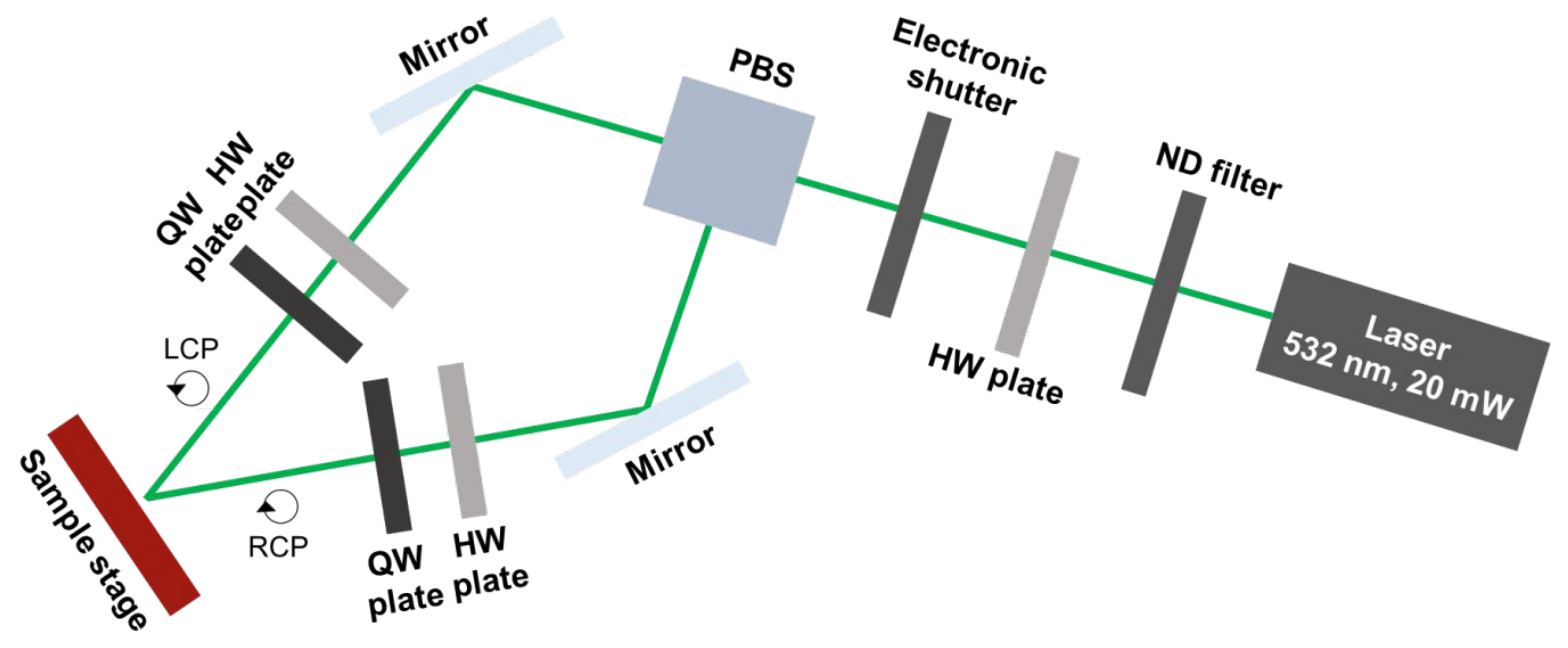

Fig. S1. Schematic illustration of the optical setup for the interference light irradiation (ND filter: neutral density filter, HW plate: half-wave plate, QW plate: quarter-wave plate, PBS: polarizing beam splitter). 
Poly (disperse orange) 3

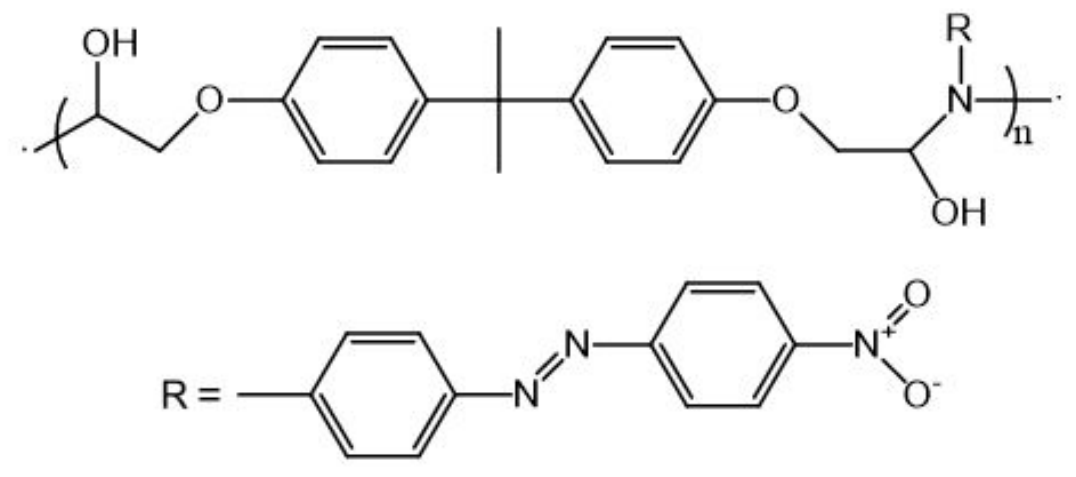

Fig. S2. Chemical structure of poly (disperse orange) 3 (PDO3). 
[ pristine pillar ]

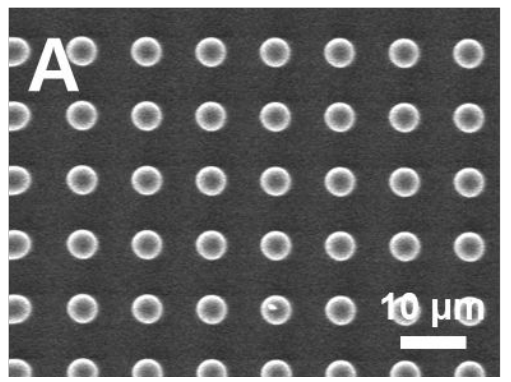

[ linear polarization]

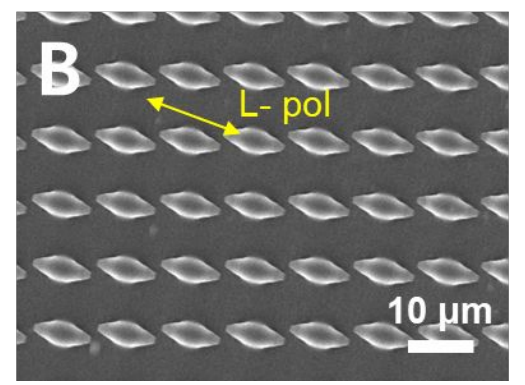

[ circular polarization]

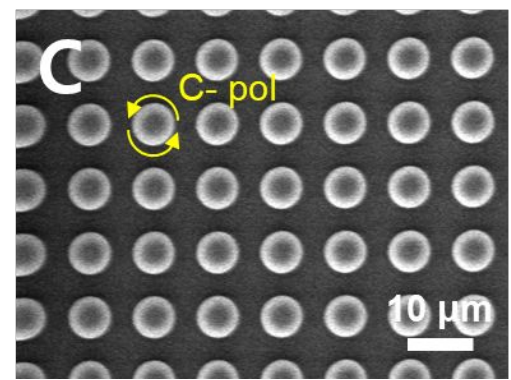

Fig. S3. Top-view SEM images of the (A) pristine pillar array and (B,C) photo-reconfigured pillar arrays by (B) linearly polarized and (C) circularly polarized light irradiation. (Scale bars $=20 \mu \mathrm{m})$ 
A

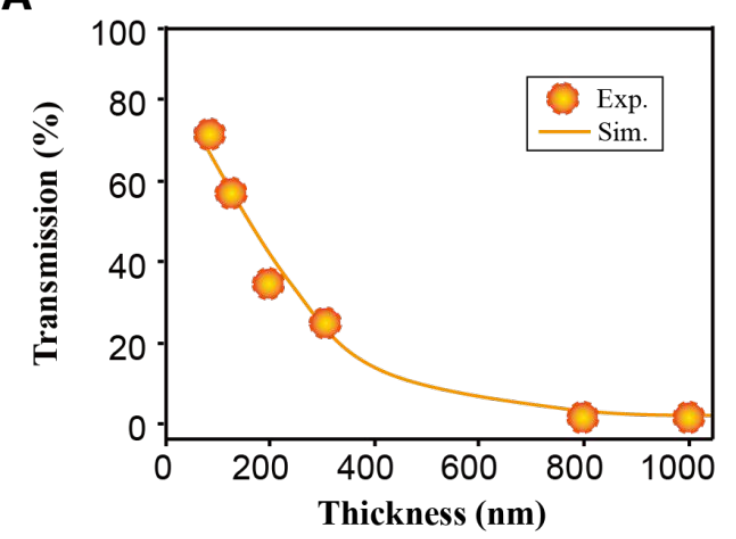

B

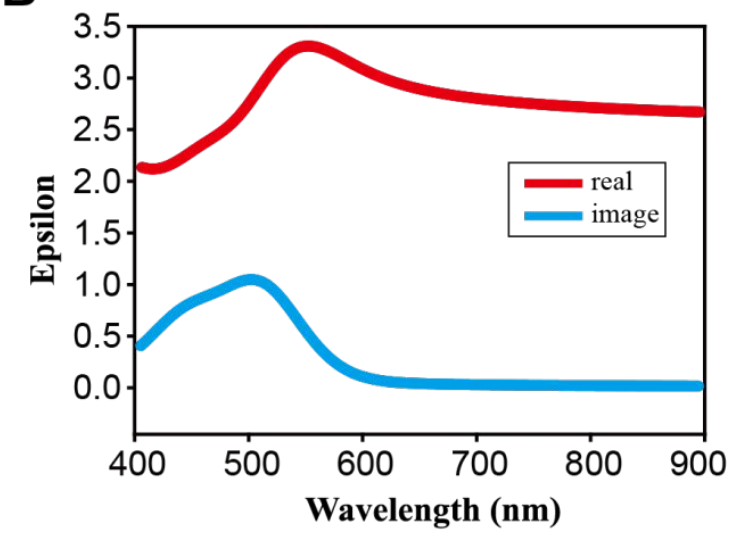

Fig. S4. (A) Plot of the transmission for the PDO3 azopolymer films as a function of the film thickness. The calculated transmission values from the Finite-Different Time-Domain (FDTD) simulations are compared. (B) The permittivity of PDO3 used for the simulations, which was experimentally measured by ellipsometry (EP3, Nanofilm). 

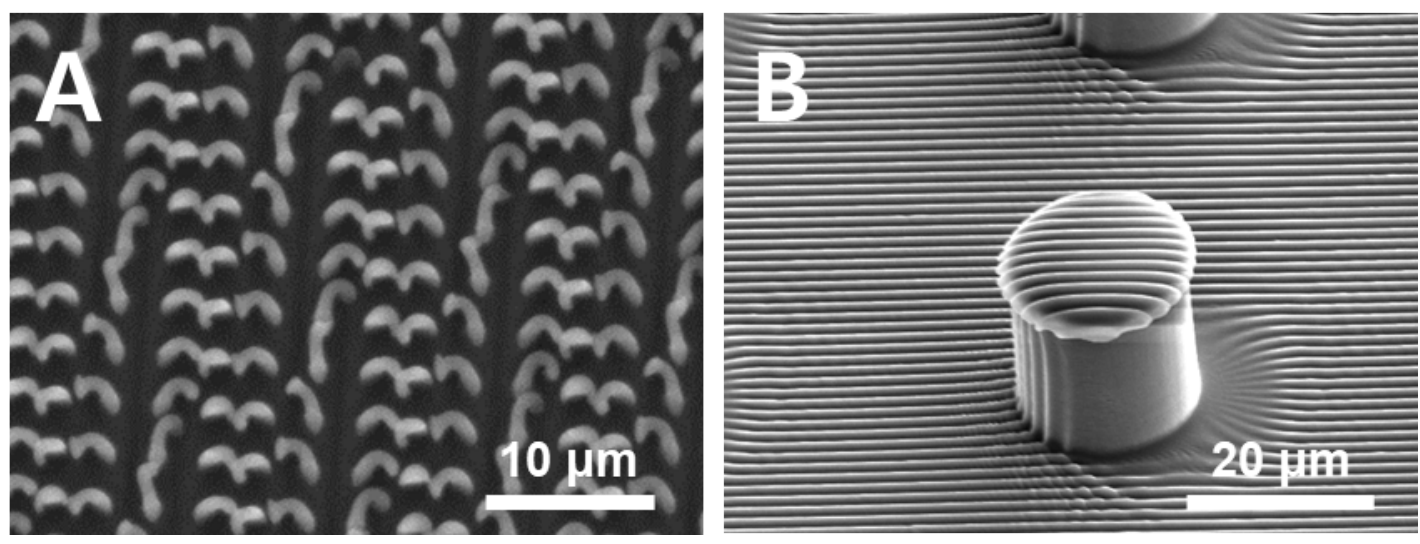

Fig. S5. SEM images of the slant interference light-irradiated pillar arrays with a pillar diameter of (A) $1 \mu \mathrm{m}$ and (B) $20 \mu \mathrm{m}$. The light irradiation conditions (t, $\theta, \varphi, \psi=5 \mathrm{~min}, 12^{\circ}$, $15^{\circ}, 0^{\circ}$, respectively) were identical for the two pillars. The wavelength of the polarization distribution $(\lambda)$ was $2.5 \mu \mathrm{m}$ at an interfenece angle of $12^{\circ}$. Because the dimater of the smaller pillar was smaller than $\lambda$, they were collapsed and bent. The bending directions dictated the polarization distribution (A). For the larger pillar, the integrity of the pillar structure was sustained; however, only the skins were reconfigured not yielding an asymmetric riblet geometry because the pillar diameter was much larger than $\lambda(B)$. 

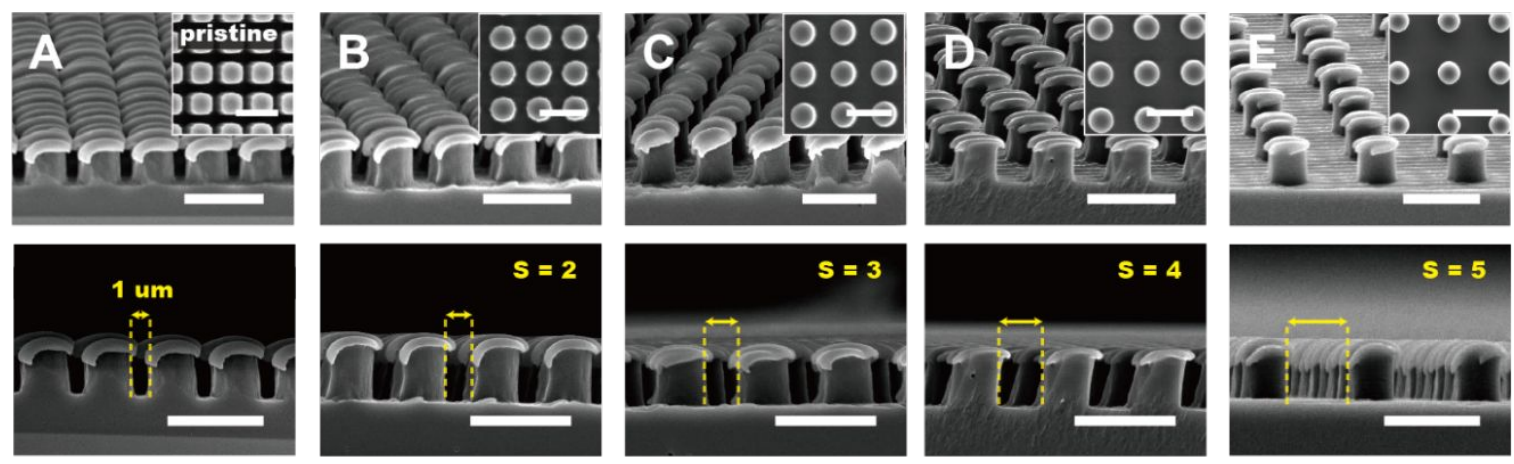

Fig. S6. Control of the areal denticle density for the shark skin-mimetic structure. SEM sideview and cross-sectional images of the denticle structures with five different spacings (S) of (A) 1, (B) 2, (C) 3, (D) 4 and (E) $5 \mu \mathrm{m}$ (Inset: corresponding pristine pillar array). 


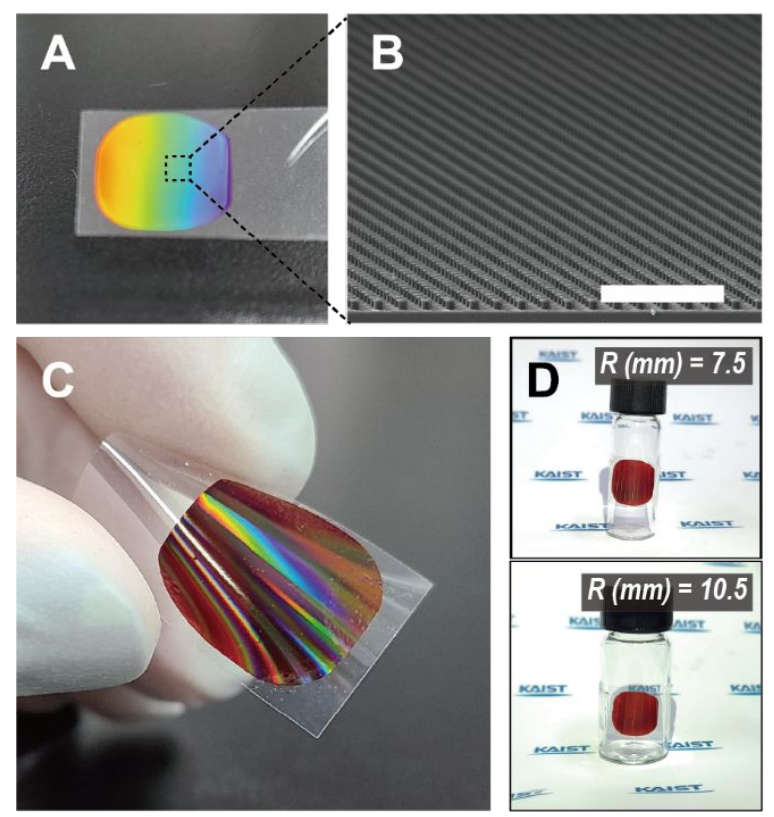

Fig. S7. Large-area fabrication and flexible shark skin-mimetic surface by irradiationbased fabrication. (A) Photograph and (B) SEM image of a large area $\left(3 \times 3 \mathrm{~cm}^{2}\right)$ shark skin-mimetic denticle surface. (C) Photographs showing the flexibility of the low drag surface formed on a polyethylene terephthalate (PET) film. (D) Bending of the film at a radius of curvature of 7.5 or $10.5 \mathrm{~mm}$ (Scale bars: $50 \mu \mathrm{m}$ ). 


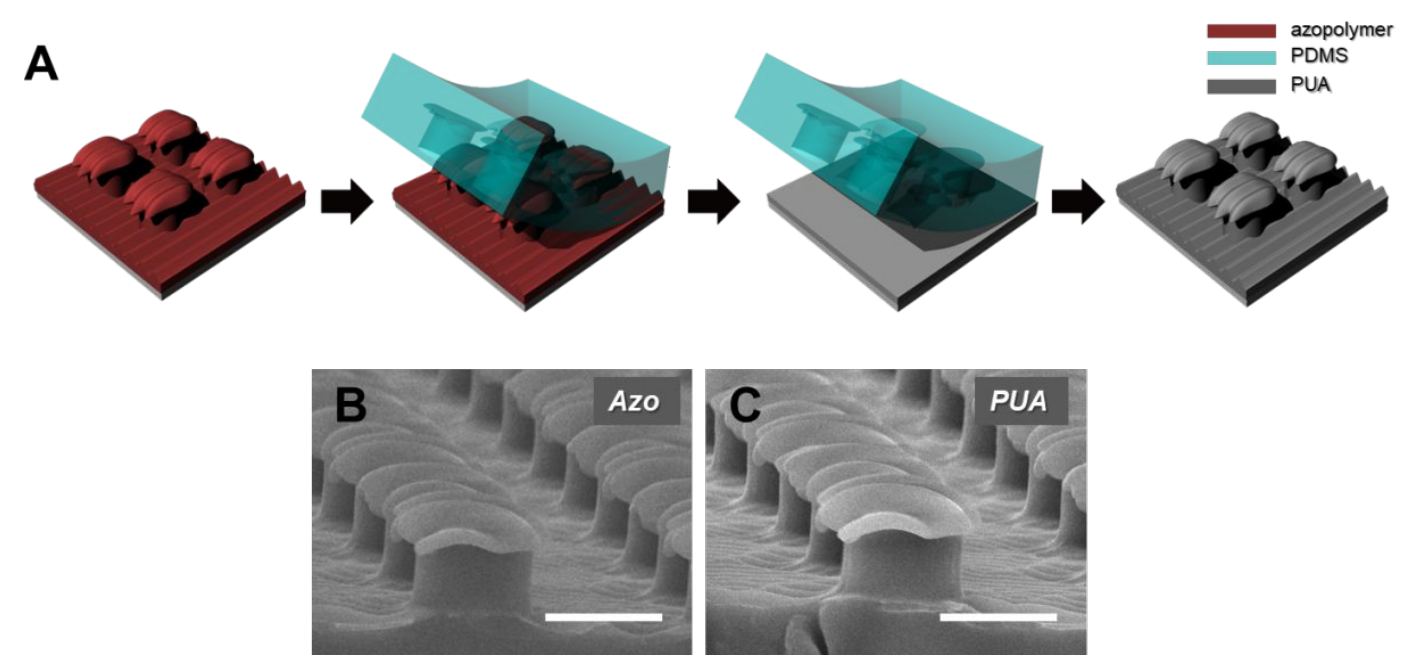

Fig. S8. (A) Schematic illustration of the shark skin-mimetic denticle structure transfer procedure. The mixture of prepolymer and a cross-linker (Sylgard 184, Dow Corning, Midland, MI ) at a weight ratio 10:1 was gently poured onto bent pillar surface and cured at $70{ }^{\circ} \mathrm{C}$ for $5 \mathrm{~h}$. The PDMS mold was then released from the azopolymer surface. A drop of PUA was spread on glass or PET substrate. The PDMS mold was then placed on the substrate. After PUA was cured by UV irradiation, the PDMS mold was gently removed from the substrate, yielding the denticle structures of PUA. (B) SEM image of pristine denticle structures of azopolymer. (C) SEM image of a tranferred denticle structure of PUA (scale bars: $5 \mu \mathrm{m}$ ). The light-designed denticle structure of azopolymer was perfectly transferred to the PUA. 

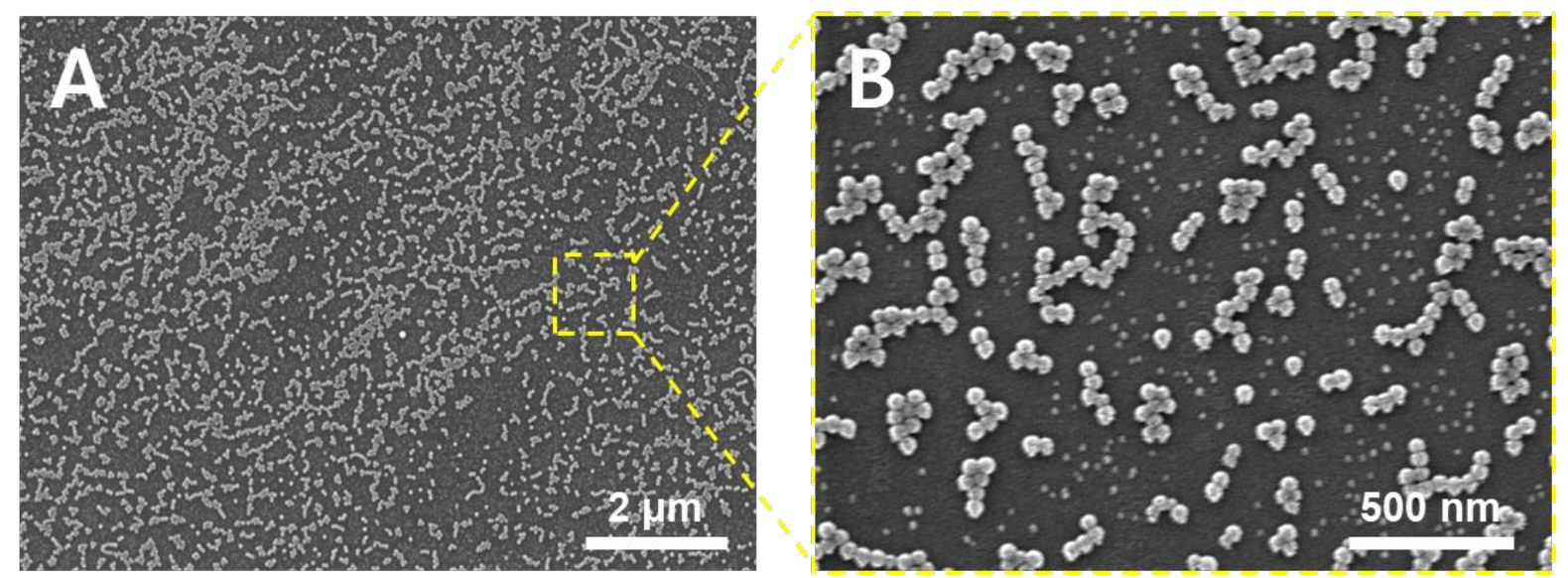

Fig. S9. (A) A SEM image and (B) its magnified image of the PS beads drop-coated on the PDO3 film. The diameter of the PS bead was $50 \mathrm{~nm}$. 

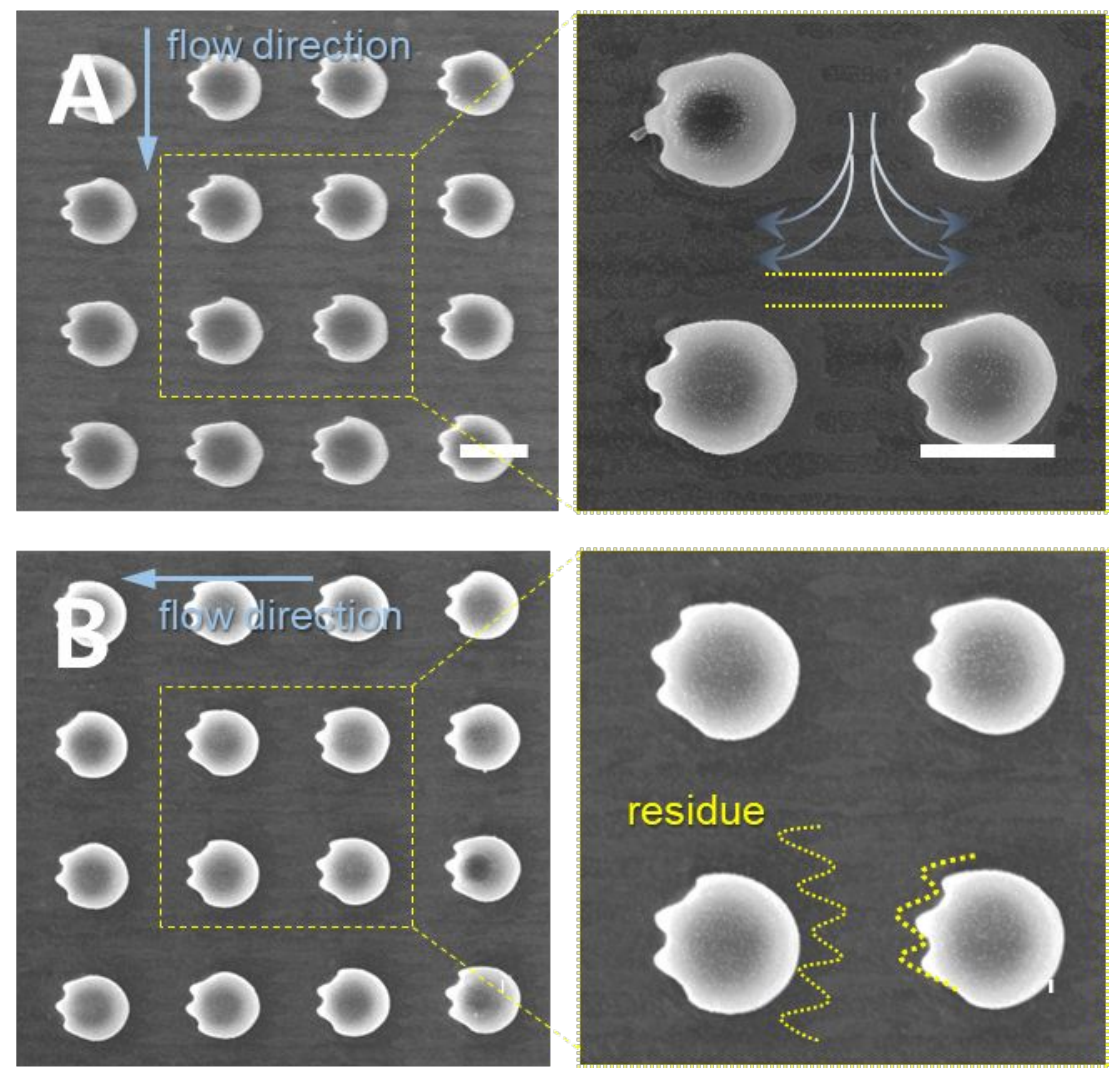

Fig. S10. SEM images of the PS bead residues after flowing a water suspension of PS beads over the shark skin-mimic surfaces in the (A) cross- and (B) reverse-direction (The light irradiation conditions for the denticle structure: $\left.(\mathrm{t}, \theta, \varphi, \psi)=\left(3 \mathrm{~min}, 12^{\circ}, 15^{\circ}, 0^{\circ}\right)\right)($ Scale bars: $5 \mu \mathrm{m})$. 